\title{
Anabases
}

\section{Pascal PAYEN, Évelyne SCHEID-TISSINIER (éd.), Anthropologie de l'Antiquité. Anciens objets, nouvelles approches}

\section{Carlamaria Lucci}

\section{(2) OpenEdition}

\section{Journals}

Édition électronique

URL : http://journals.openedition.org/anabases/5361

DOI : 10.4000/anabases.5361

ISSN : 2256-9421

\section{Éditeur}

E.R.A.S.M.E.

\section{Édition imprimée}

Date de publication : 1 avril 2015

Pagination : 283-285

ISSN : 1774-4296

\section{Référence électronique}

Carlamaria Lucci, «Pascal pAyen, Évelyne scheid-tissinier (éd.), Anthropologie de l'Antiquité. Anciens objets, nouvelles approches », Anabases [En ligne], 21 | 2015, mis en ligne le 01 avril 2015, consulté le 22 septembre 2020. URL : http://journals.openedition.org/anabases/5361 ; DOI : https://doi.org/ 10.4000/anabases.5361

Ce document a été généré automatiquement le 22 septembre 2020

(c) Anabases 


\title{
Pascal PAYEN, Évelyne SCHEID-TISSINIER (éd.), Anthropologie de l'Antiquité. Anciens objets, nouvelles approches
}

\author{
Carlamaria Lucci
}

\section{RÉFÉRENCE}

Pascal PAYEN, Évelyne SCHEID-TISSINIER (éd.), Anthropologie de l'Antiquité. Anciens objets, nouvelles approches, Turnhout, Brepols Publishers, 2012, $441 \mathrm{p}$.

80 euros / ISBN 978-2-503-54697-1

1 Structuré sous la forme d'un recueil d'essais, ce volume collectif est le premier d'une nouvelle série, consacrée par ses directeurs (C. Bonnet et P. Payen) à La Traversée des Frontières. Si l'œuvre de Jean-Pierre Vernant fait l'objet de nombreuses références, ce n'est pas le seul héritage auquel les auteurs des contributions se confrontent. L'option du grand helléniste s'était définie en faveur d'une anthropologie historique, issue de l'échange avec et entre ses deux maitres : le psychologue Ignace Meyerson et l'helléniste, de formation durkheimienne, Louis Gernet (voir R. Di Donato, Per un'antropologia storica del mondo antico, Firenze 1990 et Per una storia culturale dell'antico, I-II, Pisa 2013). Les auteurs du volume, rattachés pour une partie au centre de recherches que Vernant avait fondé à Paris (aujourd'hui ANHIMA), réfléchissent plutôt aux conditions de possibilité d'une Anthropologie de l'Antiquité. Le manque de qualificatif est ici en rapport avec la pluralité et la diversité réciproque des approches, qu'ils proposent et pratiquent, en relation avec un champ d'études plus large (le monde grec et/ou romain).

2 En ce qui concerne l'histoire de la culture, l'importance de la psychologie historique est reconnue par A. Wittenburg (p. 336), de l'université de Picardie, ainsi que par A. GrandClément (PLH-ERASME Toulouse). Celle-ci assume les fruits de la collaboration entre Meyerson et Gernet comme l'un des points de départ pour son essai sur la perception 
des couleurs en Grèce ancienne (p. 242). La fonction paradigmatique qu'elle attribue aux travaux du médiéviste Michel Pastoureau (p.240), est néanmoins significative d'une tendance que C.Bonnet (PLh-ERASMe Toulouse) et P. Schmitt Pantel (ANHIMA) rendent explicite (p.106 et 127). L'approche que chacune des deux chercheuses théorise ne se superpose pas mécaniquement à celle de Vernant. En écho avec A. Burguière (Dictionnaire des Sciences Historiques, Paris 1980), elles entendent, par anthropologie historique, le produit d'un renouvellement intrinsèque à l'histoire comme discipline académique (notamment l'histoire des Annales), à travers les apports de l'anthropologie sociale (surtout de Lévi-Strauss) et culturelle (anglo-américaine). Ce n'est pas un hasard que P. Schmitt Pantel ait été élève de Pierre Vidal-Naquet, historien de formation qui a croisé le parcours, intellectuel et humain, de Vernant. Dans une perspective analogue à celle de P.S.P., ancrée dans les limites disciplinaires de l'histoire, V. Azoulay et V. Sebillotte-Cuchet (ANHIMA) intègrent les apports, respectivement, de l'anthropologie culturelle et des gender studies. Il s'agit, pour l'un, de relire Mauss et Gernet, afin de renouveler le thème classique du don en Grèce ancienne, pour l'autre, de fonder une anthropologie du genre (comme notion historiquement construite), à travers la critique des travaux de Nicole Loraux sur la différence des sexes.

La perspective de P. Payen (PLH-ERASME Toulouse) est symétrique : par son approche du monde grec elle relève moins d'une anthropologie historique que d'une histoire structurale contaminant la longue durée de Braudel et les invariants de Lévi-Strauss (p. 207). La guerre des cités lui apparaît comme un phénomène de conjoncture dont l'analyse peut amener à l'identification de tendances sous-jacentes, telles le rapport polis-stasis, que Nicole Loraux avait brillamment esquissé. Avec É. Scheid-Tissinier (ANHIMA), auteur de l'introduction au volume et d'un essai sur les émotions en Grèce ancienne, l'approche historique, au sens étroit du terme, laisse place à une approche, linguistique et sémantique, dont elle a montré l'efficacité dans son ouvrage de 1994 (Les usages du don chez Homère. Vocabulaire et pratique). Son intérêt (p. 9-10) glisse vers le Gernet sociologue des années 1910, ainsi que vers l'identification d'une ligne de continuité avec le Vernant du mythe des races (années 1960).

Du côté des spécialistes du monde romain, l'emploi de la notion d'anthropologie n'est pas moins varié. Les approches mobilisées par T. Derks (Amsterdam) et N. Purcell (Oxford) montrent des points communs avec celle que théorise P. Schmitt Pantel. Dans son projet d'archéologie historico-anthropologique, Derks situe sa source d'inspiration dans l'École des Annales (p. 49); Purcell utilise indifféremment les notions d'anthropologie historique (p. 82), d'histoire anthropologique (p. 83), d'anthropologie sociale (p. 96, 98). Si, par conséquent, les références à Mauss, Gernet, Vernant, Finley (pour le monde anglo-saxon) ne manquent pas, le pivot de la démarche interprétative consiste en une contamination directe de données documentaires et de grilles catégoriales tirées de l'anthropologie culturelle. Tel est le sens des références de Derks, dans son étude sur les rites de passage dans la Gaule romaine, aux modèles de tripartition initiatique introduits par A. Van Gennep et V. Turner (p.51-52). Tel est, encore, le sens des références de Purcell, dans son étude sur les représentations et pratiques d'achat et vente dans les mondes grec et romain, à Geertz (p. 93) et Appadurai (p. 88).

5 Représentant d'une anthropologie du monde ancien spécifiquement siennoise, M. Bettini transpose à un témoignage de Plaute (Aulularia 1-36) sur le Lar Familiaris la 
distinction interprétative, introduite dans les sciences sociales anglo-américaines, entre les points de vue étique et émique. Il montre en quelle mesure la philologie se fait instrument privilégié du passage des catégories de l'observateur (moderne) aux catégories des acteurs d'un monde révolu (les formes des représentations de la parenté des anciens Romains).

6 À signaler, enfin, l'ouverture de chemins nouveaux dans le cadre du centre ANHIMA : tel est le cas de l'anthropologie de l'écriture pratiquée par E. Valette. L'identification de la fonction rituelle de l'écriture funéraire, en tension avec l'écriture littéraire de l'élégie romaine, montrent les potentialités d'une approche redevable autant aux études classiques de J.L. Austin (sur les actes de paroles) qu'aux études de C.Calame et F. Dupont (pareillement actifs au Centre) sur les dimensions discursives/pragmatiques de la poésie ancienne.

\section{AUTEURS}

\section{CARLAMARIA LUCCI}

Dipartimento di Filologia Classica, Università di Pisa carlamarialucci@interfree.it 